Full Length Article

\title{
Involvement of Endogenous Abscisic Acid and Cytokinin in Photosynthetic Performance of Different Stay Green Inbred Lines of Maize under Drought
}

\author{
Yufei Zhou $^{*}$, Dequan Wang ${ }^{1}$, Na Wang ${ }^{1}$, Jialin Yu², Yitao Wang ${ }^{3}$, Qi Wu ${ }^{1}$, Wenjuan Xu ${ }^{1}$ and Ruidong Huang ${ }^{*}$ \\ ${ }^{1}$ College of Agronomy, Shenyang Agricultural University, Shenyang, Liaoning Province 110866, People's Republic of China \\ ${ }^{2}$ Department of Crop and Soil Sciences, University of Georgia, 1109 Experiment Street, Griffin, GA 30223, USA \\ ${ }^{3}$ Agro-environmental protection and supervision center, Liaoning Province 110034, People's Republic of China \\ *For corresponding author: zhouyufei2002@aliyun.com; r_huang@126.com
}

\begin{abstract}
Stay green, a characteristic of delaying leaf senescence, is desirable for maintaining photosynthetic activities, particularly under drought conditions. This study investigated the endogenous abscisic acid (ABA) and cytokinin (CTK) in maize plants and their potential relations with photosynthetic activities during late growth stage under drought stress. Two maize inbred lines, including stay green inbred line (Dan598) and non-stay green inbred line (Huangzao4), were planted in pots. Plants were treated with non-drought stress (75-80\% of the field moisture capacity) and drought stress (45-50\% of the field moisture capacity), which were maintained by using soil moisture sensor at anthesis or grain filling stage, respectively. The green leaf area per plant, chlorophyll contents (Chl), photosynthetic rate (Pn), and stomatal conductance (Gs) of Huangzao4 decreased more considerably compared to those of Dan598 under drought stress. Maize plants with stay green had greater photosynthetic performance than non-stay green under drought conditions. In addition, chlorophyll fluorescence parameters including maximal photochemical efficiency $(\mathrm{Fv} / \mathrm{Fm})$, photochemical quenching $(\mathrm{qL})$, and electron transport rate (ETR) of both maize inbred lines had the same decreasing trend as compared to their respective Pn. Drought reduced photosystem II (PS II) original fluorescence (Fo) in Dan598 but increased Fo in Huangzao4. Drought stress had substantially increased ABA but reduced zeatin riboside $(\mathrm{ZR})$ contents in the leaves and roots. Moreover, ABA content was negatively correlated with Pn, while ZR and ZR/ABA were positively correlated with Chl, Pn, Gs, Fv/Fm, qL, and ETR. Overall, the results suggested that both ABA and CTK were involved in controlling photosynthetic performance, and greater photosynthetic activity of stay green maize could be attributed to higher ZR and ZR/ABA when subjected to drought. (C) 2016 Friends Science Publishers
\end{abstract}

Keywords: Abscisic acid; Drought stress; Maize; Photosynthesis; Stay green; Zeatin riboside

\section{Introduction}

The variation of precipitation in recent years as a result of climate change often results in drought stress, which negatively influences the maize production (Ji et al., 2012). In some major maize-producing areas such as the maize belt of China, maize yield underwent $9.3-35.1 \%$ loss due to the drought stress (Wang and Li, 2010). Thus, drought tolerance is becoming an increasing important target in maize breeding programs. One solution is to identify the traits associated with drought tolerance (Lu et al., 2011) and utilize superior germplasm resources to further improve drought tolerance and yield stability.

Stay green is considered as a desirable trait associated with postanthesis drought tolerance (Borrell et al., 2000; Nawaz et al., 2013). The most distinguishing phenotype of stay green plant is delaying leaf senescence. Plants with stay green trait exhibit notable drought tolerance and resistance to premature leaf senescence, pests, and lodging during the late growth stage when plants suffered drought stress (Rosenow et al., 1983; Tenkouano et al., 1993; Walulu et al., 1994; Borrell et al., 2000). In maize breeding programs, enhanced stay-green traits have been documented to be an important approach to improve yield under drought stress (Campos et al., 2004). However, research is limited on the understanding of physiological processes associated with this stay green trait in maize than in other cereals including sorghum (Sorghum bicolor L. Moench). In sorghum, Huang et al. (2009) reported the effective antioxidant metabolism related to stay green trait improved the resistance to premature senescence under drought stress. The authors also noted that sorghum with stay green trait maintained the high photosynthetic capacity and chlorophyll content under drought stress.

The inhibition of photosynthesis as a consequence of drought stress results from the disturbance of photosynthetic

To cite this paper: Zhou, Y., D. Wang, N. Wang, J. Yu, Y. Wang, Q. Wu, W. Xu and R. Huang, 2016. Involvement of endogenous abscisic acid and cytokinin in photosynthetic performance of different stay green inbred lines of maize under drought. Int. J. Agric. Biol., 18: 1067-1074 
process. Drought triggers an imbalance between the generation and utilization of electrons in photosystem II (PSII), resulting in changes of quantum transport in PSII (Farooq et al., 2009; Zhang et al., 2011). Consequently, reactive oxygen species are produced in PSII, leading to the photoperoxidation of thylakoid and other cell membrane lipids (Demmig-Adams and Adams, 1992; Ladjal et al., 2000). Endogenous hormones play crucial roles in plant physiological processes during the adaptation of adverse environments. Abscisic acid (ABA) is an essential regulator in integrating plant responses to drought (Zhang et al., 2006). Under drought stress, ABA protects plant photosynthesis by enhancing the activity of antioxidant enzymes (Li et al., 2010) and xanthophylls cycle (Jia and $\mathrm{Lu}, 2003)$. Cytokinins (CTK) is also a major endogenous hormone involved in plant growth and development. Moreover, CTK is found to inhibit ABA-induced stomatal closure (Wang et al., 2004; Tanaka et al., 2006). Up to now, there has been limited research on the photosynthetic performance and the associated activity of ABA and CTK in stay green maize to drought stress. We hypothesize that the endogenous contents of ABA and CTK are involved in controlling photosynthetic performance during drought stress. Therefore, the objective of this research was to investigate the photosynthetic performance of two different stay green maize and changes in ABA and CTK in leaves and roots subjected to drought at anthesis or grain filling stage, respectively and determine whether and how the changes of $\mathrm{ABA}$ and $\mathrm{CTK}$ were related with photosynthetic performance. The results possess the potential for better understanding the adapted mechanisms of stay green maize under drought.

\section{Materials and Methods}

\section{Plant Materials and Treatments}

Two separate experiments were carried out in 2010 and 2011 in the experimental station of Shenyang Agricultural University, Shenyang, China. Stay green maize inbred line Dan598 and non-stay maize inbred line Huangzao4 as materials were planted in plastic pots measuring $30 \mathrm{~cm}$ depth and $33 \mathrm{~cm}$ diameter. These pots were filled with $17 \mathrm{~kg}$ field soil that was collected from the $20 \mathrm{~cm}$ surface layer in the arable field. The soil $(\mathrm{pH} 6.68$; organic matter $35.03 \mathrm{~g}$ $\mathrm{kg}^{-1}$; alkali hydrolysable $\mathrm{N}$, available $\mathrm{P}$, and available $\mathrm{K}$ $120.42,6.86,116.21 \mathrm{mg} \mathrm{kg}^{-1}$, respectively) used in this experiment was air dried and passed through a $3 \mathrm{~mm}$ sieve to exclude the clods and stubble. The pots were arranged in a completely randomized design. There were 48 pots per treatment and each treatment replicated three times.

Three seeds were sown in each pot on May 8 in 2010 and 2011, respectively. Seeds were selected based on size and uniformity. Seedlings were thinned to one plant per pot when reached fifth-leaf stage. According to local cultivation, diammonium phosphate at $150 \mathrm{~kg} \mathrm{ha}^{-1}$ was applied as basal fertilizer. At the stage of stem elongation, each pot received $3.3 \mathrm{~g}$ urea that equivalent to $20 \mathrm{~kg} \mathrm{ha}^{-1}$ for promoting plant establishment.

All pots were irrigated equally before the flowering stage. Two water treatments, including well-watered (75$80 \%$ of field moisture capacity) and water deficit (45-50\% of field moisture capacity) were imposed at either flowering stage (Initiated on July 29 in 2010 and July 31 in 2011, respectively) or filling stage at $20 \mathrm{~d}$ from anthesis (initiated on August 18 in 2010 and August 20 in 2011, respectively). The whole soil profile was allowed to dry down to the 45$50 \%$ of the field moisture capacity and used as drought stress treatment. The soil moisture content was maintained by using soil moisture sensor (HH2/ML2X, Delta-T, UK). The period of drought stress lasted $7 \mathrm{~d}$ and then the stressed plants were re-watered normally till maturity. The pots were protected by a mobile rain shelter from the rain. Parameters and samples were obtained timely after water stress period.

\section{Green Leaf Area Investigation}

Green leaf area per plant is the sum of all green leaves areas of a plant. A green leaf area is calculated as leaf length $\times$ leaf width $\times 0.75$.

\section{Chlorophyll Determination}

Chlorophyll samples were extracted from fresh ear leaf sections $(0.1 \mathrm{~g})$ with $10 \mathrm{~mL} 80 \%$ acetone, and chlorophyll contents were quantified according to Arnon (1949).

\section{Photosynthetic Parameters and Chlorophyll Fluorescence}

Photosynthetic rate (Pn) and stomatal conductance (Gs) were determined on the ear leaf (15 replications per treatment) between 09:30 and 11:00 AM using a portable photosynthesis system (Li-6400, Li-Cor Lincoln, NE, USA). Chlorophyll fluorescence parameters were measured on the same leaf as the measurement of photosynthetic parameters with a pulse-amplitude-modulation system (Junior-pam fluorometer, Walz-Effeltrich, Germany). Sampled leaves were acclimated in dark for $30 \mathrm{~min}$ and then exposed to a weak modulated pulse $\left(<0.1 \mu \mathrm{mol} \mathrm{m} \mathrm{m}^{-2} \mathrm{~s}^{-1}\right)$, followed by the exposure to a $0.8 \mathrm{~s}$ saturating flash of light $\left(10,000 \mu \mathrm{mol} \mathrm{m} \mathrm{m}^{-2} \mathrm{~s}^{-1}\right)$. The following fluorescence parameters used in measurements were adapted from van Kooten and Snel (1990): maximal PS II photochemical efficiency (Fv/Fm), PS II original fluorescence (Fo), photochemical quenching $(\mathrm{qL})$, and electron transport rate (ETR).

\section{Hormonal Determination}

Samples of the ear leaves and roots from different plants for each treatment were selected and frozen in liquid nitrogen for $1 \mathrm{~min}$ and then stored at $-80^{\circ} \mathrm{C}$ until $\mathrm{ABA}$ and zeatin riboside (ZR) extraction. Samples of $0.5 \mathrm{~g}$ of the frozen leaves or roots were ground in a mortar at $0{ }^{\circ} \mathrm{C}$ with $4 \mathrm{~mL}$ $80 \%(\mathrm{v} / \mathrm{v})$ methanol containing $1 \mathrm{mmol} \mathrm{L}^{-1}$ butylated 
hydroxytoluence as extraction medium. The extract was incubated at $4{ }^{\circ} \mathrm{C}$ for $4 \mathrm{~h}$ and centrifuged at $4000 \mathrm{~g}$ for 15 $\min$ at $4{ }^{\circ} \mathrm{C}$. The supernatants were purified by passing through $\mathrm{C} 18$ columns. Then the hormone fractions were dried by $\mathrm{N}_{2}$, and dissolved in $2 \mathrm{~mL}$ phosphate buffer saline (PBS) containing $1 \%(\mathrm{v} / \mathrm{v})$ Tween 20 and $1 \%(\mathrm{w} / \mathrm{v})$ gelatin for analysis. The ABA and ZR contents were analyzed by an enzyme-linked immunosorbent assay (ELISA) using assay kits (China Agricultural University). The methods for extraction and quantification of $\mathrm{ABA}$ and $\mathrm{ZR}$ were described by Yang et al. (2001).

\section{Statistical Analyses}

Experiments were conducted as a complete randomized design with three replications. Data were subjected to oneway analysis of variance in SPSS 18.0. Treatment means were separated with Fisher's protected least significant difference (LSD) at $\alpha=0.05$. The results were presented as the mean \pm standard deviation (SD). Experiment by treatment interaction was not detected and thus, the data were pooled across experimental runs for analysis.

\section{Results}

\section{Green Leaf Area per Plant}

Drought stress markedly $(p \leq 0.05)$ decreased the green leaf area per plant in both maize lines (Fig. 1). The green leaf area per plant in Huangzao 4 was decreased by $27.5 \%$ and $34.0 \%$, respectively compared with well-watered plants after drought stress occurred at flowering stage and filling stage. However, that of Dan598 decreased by $9.6 \%$ and $15.2 \%$, respectively.

\section{Chlorophyll Contents}

Chlorophyll contents (Chl) in both Huangzao4 and Dan598 were significantly decreased when plants suffered drought stress (Fig. 1). However, the decreased extent in Huangzao4 was larger than that in Dan598. Compared with wellwatered plants, the drought stress notably $(p \leq 0.05)$ reduced the chlorophyll content in Huangzao4 and Dan598 by $31.6 \%$ and $14.3 \%$ at flowering stage and $41.2 \%$ and $20.5 \%$ at filling stage, respectively. At the filling stage, the declined extent of chlorophyll content in Huangzao4 was obviously higher than that in Dan598, indicating that the stay green could delay the degradation of chlorophyll content at late growth stage.

\section{Gas-exchange Parameters}

Photosynthetic rate $(\mathrm{Pn})$ exhibited a notable reduction $(p \leq 0.05)$ under drought stress (Fig. 2). Compared with wellwatered treatments, Pn in Huangzao4 and Dan598 was decreased by $43.6 \%$ and $30.4 \%$ at flowering stage and $43.9 \%$ and $26.0 \%$ at filling stage respectively, suggesting

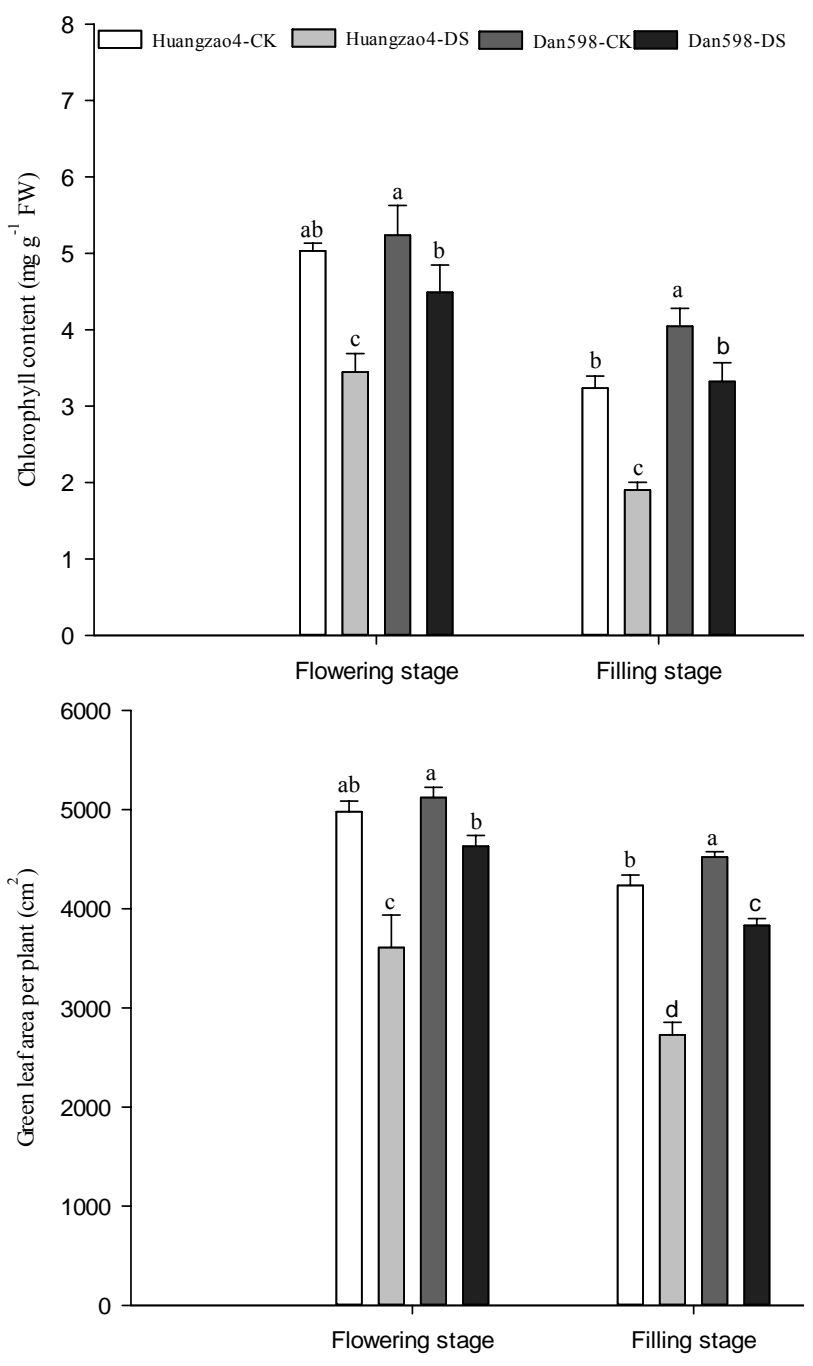

Fig. 1: Variations in chlorophyll content and green leaf area per plant of stay green maize (Dan598) versus non-stay green maize (Huangzao4) under drought stress. Vertical bars indicate standard deviations. The different letters indicate significant difference at the 0.05 probability level (LSD test) within the same stage. $\mathrm{CK}$, watered normally; DS, drought stress

that drought stress had a more negative effect on Pn of nonstay green maize than that of stay green maize.

Stomatal conductance (Gs) in both maize lines had significantly reduced by drought stress (Fig. 2). At flowering stage, Gs of drought-stressed plants reduced by $80.0 \%$ in Huangzao4 and $33.3 \%$ in Dan598, respectively. At filling stage, Gs of stray-green maize had not significantly declined under drought stress as compared to plants grown under unstressed controls. In comparison, drought stress remarkably reduced $(p \leq 0.05)$ Gs in Huangzao4.

\section{Chlorophyll Fluorescence}

Drought stress notably $(p \leq 0.05)$ enhanced PS II original 

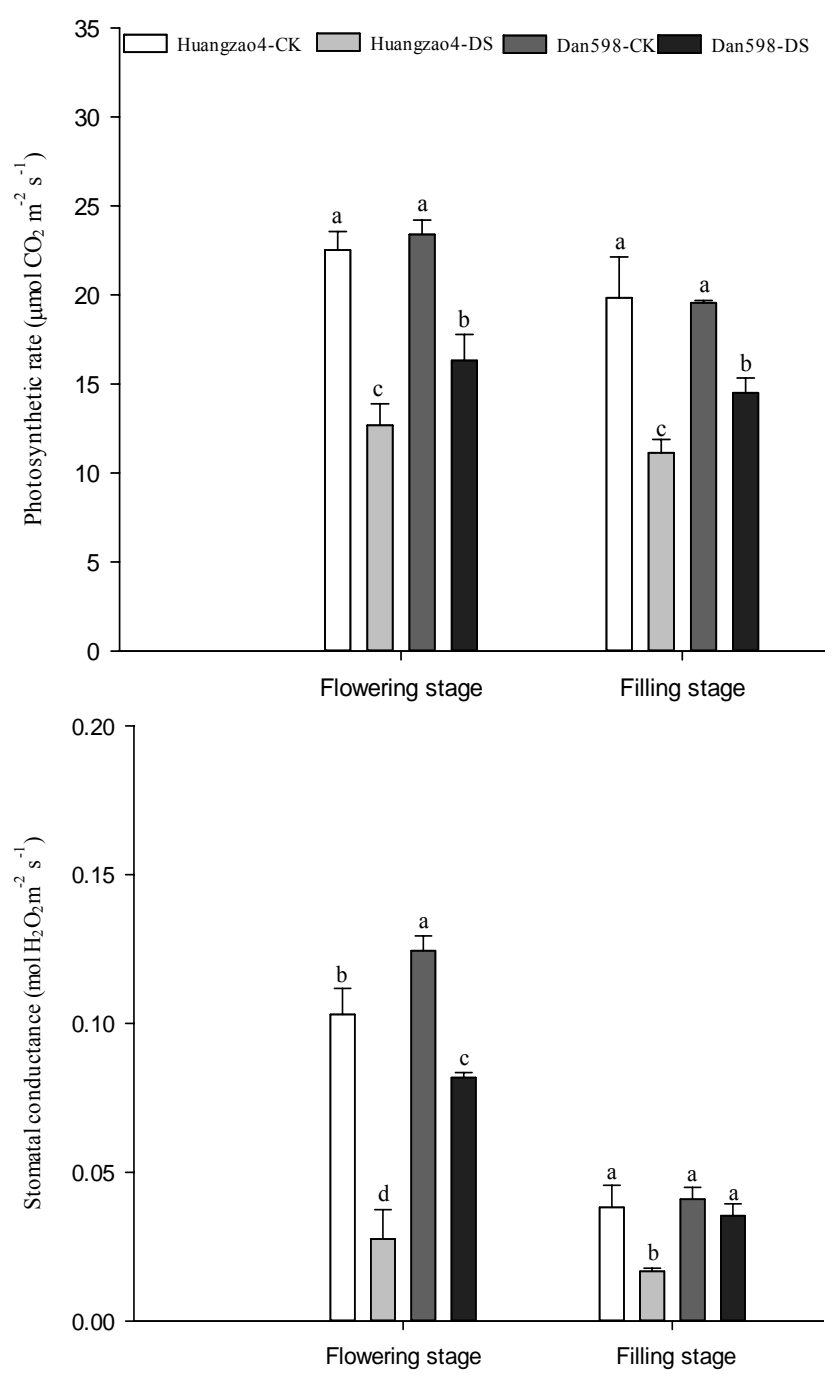

Fig. 2: Variations in photosynthetic rate $(\mathrm{Pn})$ and stomatal conductance (Gs) of stay green maize (Dan598) versus non-stay green maize (Huangzao4) under drought stress. Vertical bars indicate standard deviations. The different letters indicate significant difference at the 0.05 probability level (LSD test) within the same stage. CK, watered normally; DS, drought stress

fluorescence ( $\mathrm{Fo}$ ) in Huangzao4, whereas decreased Fo in Dan598 significantly $(p \leq 0.05)$ at flowering stage (Fig. 3). Maximal PS II photochemical efficiency $(\mathrm{Fv} / \mathrm{Fm})$ in Huangzao4 was markedly $(p \leq 0.05)$ decreased by drought stress, but there was no obvious difference of $\mathrm{Fv} / \mathrm{Fm}$ in Dan598 between drought-stressed plants and well-watered plants. Drought stress significantly $(p \leq 0.05)$ reduced photochemical quenching (qL) in both maize lines. Compared with well-watered plants, $\mathrm{qL}$ in Huangzao4 and Dan598 was decreased by $20.0 \%$ and $14.5 \%$ at flowering stage and $27.5 \%$ and $25.4 \%$ at filling stage, respectively. Changes of electron transport rate (ETR) in Huangzao4 and Dan598 under drought stress showed the similar pattern compared to $\mathrm{qL}$.

\section{Changes of Contents of ABA and ZR in Leaves}

Drought stress significantly $(p \leqslant 0.05)$ increased abscisic acid (ABA) contents of leaves in both maize lines (Fig. 4). Compared with well-watered plants, ABA contents in Huangzao 4 and Dan598 were increased by $15.6 \%$ and $38.5 \%$ at flowering stage and $20.4 \%$ and $59.2 \%$ at filling stage, respectively. The enhanced extent of ABA content in Dan598 was obviously higher than that in Huangzao4. In comparison, drought stress significantly $(p \leqslant 0.05)$ decreased ZR contents of leaves in both maize lines with a reduction of $34.1 \%$ and $37.6 \%$ for Huangzao4 and $28.9 \%$ and $30.1 \%$ for Dan598 at both flowering and filling stage, respectively.

\section{Changes of Contents of ABA and ZR in Roots}

Drought stress markedly $(p \leqslant 0.05)$ increased ABA contents of roots in both maize lines (Fig. 5). Under drought stress, ABA contents in Huangzao4 was increased by $16.9 \%$ and $9.6 \%$, respectively at flowering stage and filling stage compared with well-watered plants. However, that of Dan598 increased by $36.8 \%$ and $47.3 \%$, respectively. Contrarily, drought stress markedly $(p \leqslant 0.05)$ decreased ZR contents of roots in both maize lines with a reduction of $25.9 \%$ and $35.4 \%$ for Huangzao 4 and $26.6 \%$ and $27.9 \%$ for Dan598 at both flowering and filling stage, respectively.

\section{Relationship between Hormone Contents and Photosynthetic Parameters}

ZR content exhibited a significant positive correlation $(p \leq 0.01)$ with $\mathrm{Chl}, \mathrm{Pn}, \mathrm{Gs}, \mathrm{Fv} / \mathrm{Fm}, \mathrm{qL}$ and ETR (Table 1). ABA content was negatively correlated with $\operatorname{Pn}(p \leq 0.05)$. Similar with ZR content, ZR/ABA was positively corrected with Pn and ETR $(p \leq 0.01)$ and $\mathrm{Chl}, \mathrm{Gs}, \mathrm{Fv} / \mathrm{Fm}$ and $\mathrm{qL}$ $(p \leq 0.05)$, respectively.

\section{Discussion}

Plant leaves are vulnerable to the effects of drought. In response to drought, plants exhibit progressive wilting, yellowing, and senescence, because of reduced chlorophyll content. Genotypes containing the stay green trait can delay leaf senescence to maintain active photosynthesis than those of genotypes not containing this trait (Zhang et al., 2013; Zhou et al., 2014). Our results showed that non-stay green had a greater reduction in chlorophyll content than stay green maize under drought stress (Fig. 1). This finding is in agreement with Huang et al. (2009) who reported that chlorophyll content in stay green sorghum was less affected by drought stress compared to non-stay green sorghum. Any chlorophyll reduction may reduce plant photosynthetic capability because chlorophyll is the foundation for photosynthesis. Higher chlorophyll content in stay green 
Table 1: Relationship between hormonal contents in ear leaf and photosynthetic parameters

\begin{tabular}{lllllll}
\hline & Chl & Pn & Gs & F & Fv/Fm & EL \\
\hline ZR & $0.87 * *$ & $0.97 * *$ & $0.82 * *$ & -0.42 & $0.83 * *$ & $0.85 * *$ \\
ABA & -0.54 & $-0.72 *$ & -0.48 & 0.05 & -0.40 & -0.53 \\
ZR/ABA & $0.78^{*}$ & $0.93 * *$ & $0.74 *$ & -0.26 & $0.71 *$ & $0.77 *$ \\
\hline
\end{tabular}

ABA, abscisic acid; ZR, zeatin riboside; Chl, chlorophyll content; Pn, photosynthetic rate; Gs, stomatal conductance; Fo, PS-II original fluorescence; Fv/Fm, maximal PS-II photochemical efficiency; qL, photochemical quenching; ETR, electron transport rate

**, significant at 0.01 probability level; *, significant at 0.05 probability level
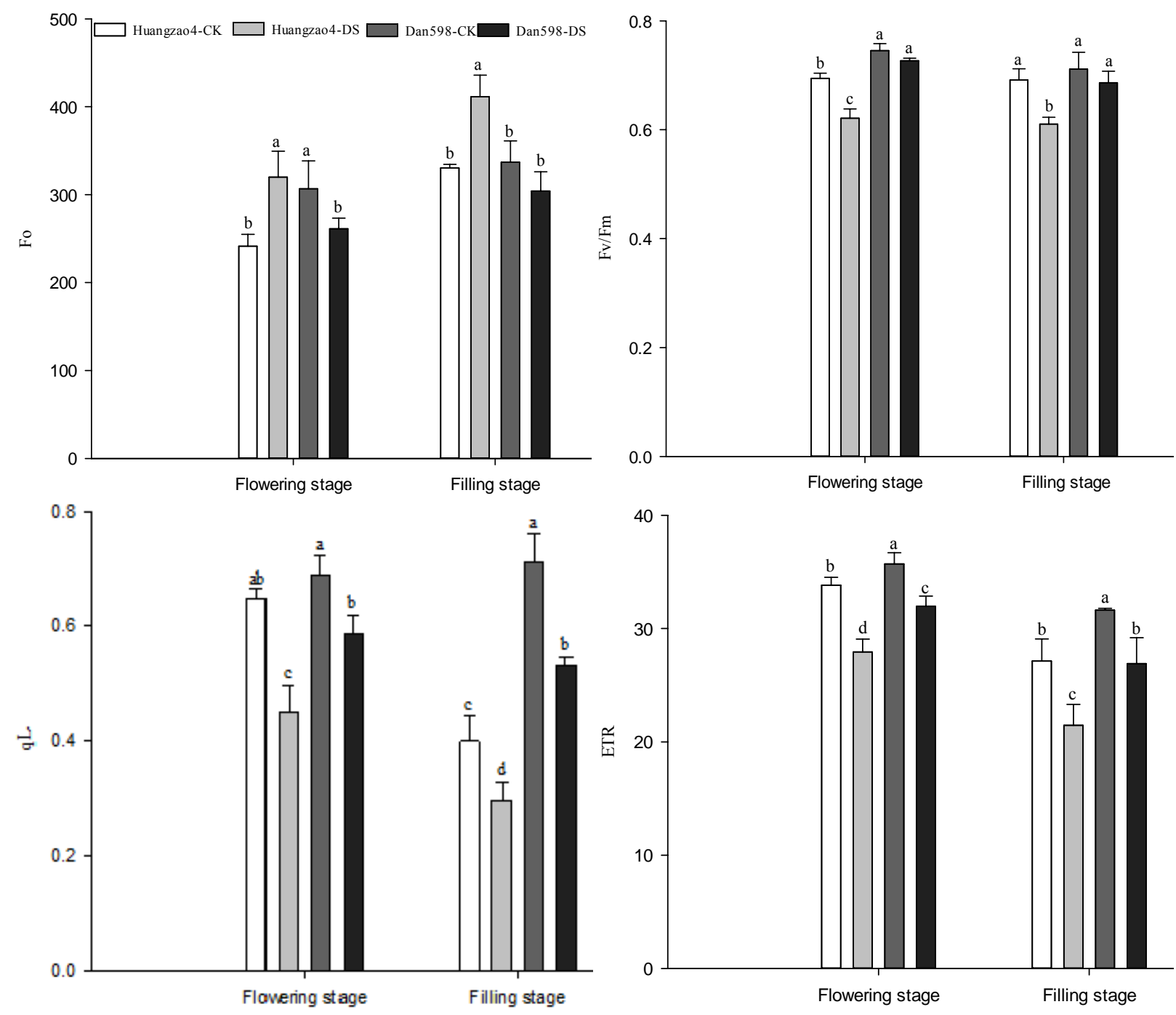

Fig. 3: Variations in maximal PS-II photochemical efficiency (Fv/Fm), PS-II original fluorescence (Fo), photochemical quenching (qL), and electron transport rate (ETR) of stay green maize (Dan598) versus non-stay green maize (Huangzao4) under drought stress. Bars indicate standard deviations. The different letters indicate significant difference at the 0.05 probability level (LSD test) within the same stage. CK, watered normally; DS, drought stress

maize may protect the photosynthesis against drought stress. We also observed in the present research that stay green maize had higher green leaf area per plant during the late growth period especially under drought, indicating that plants with stay green trait can delay leaf senescence. Overall, our results suggested that stay green maize had higher photosynthetic capability than non-stay green maize under drought conditions.

Chlorophyll fluorescence has been noted as a critical indicator of photosynthetic performance in plants (Zhang, 1999) and was widely utilized for determining the performance and viability of plants in response to drought (e.g., Faraloni et al., 2011; Mishra et al., 2012). In our study, Fv/Fm, qL and ETR were decreased under 

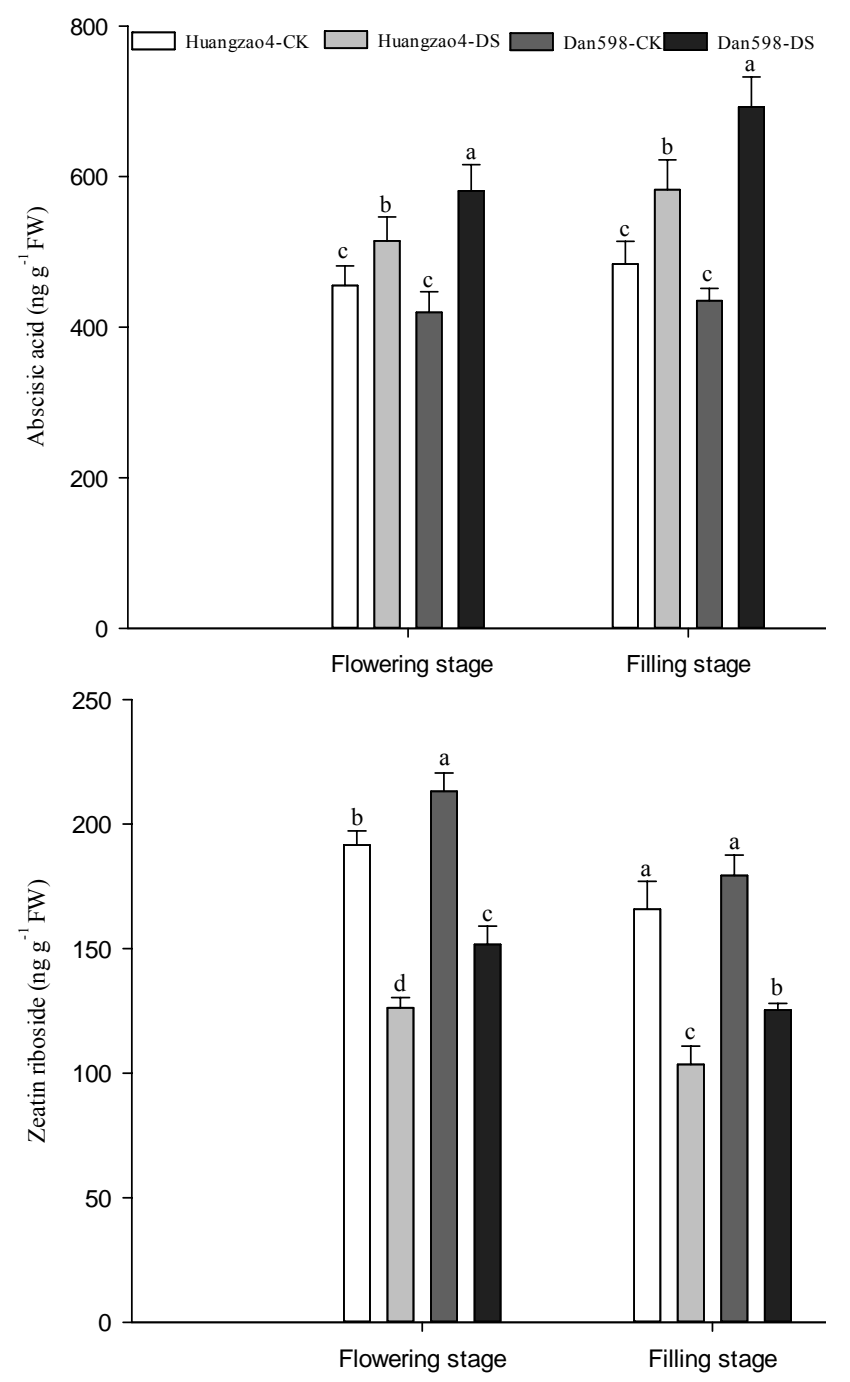

Fig. 4: Variations in abscisic acid (ABA) and zeatin riboside (ZR) in ear leaves of stay green maize (Dan598) versus non-stay green maize (Huangzao4) under drought stress. Bars indicate standard deviations. The different letters indicate significant difference at the 0.05 probability level (LSD test) within the same stage. CK, watered normally; DS, drought stress

drought stress, indicating that drought stress inhibited the photochemical activity of PS II (Fig. 3). In addition, Fv/Fm, $\mathrm{qL}$ and ETR in Huangzao4 showed a greater reduction as compared to Dan598. This implied that PS II photochemistry activities of non-stay green maize were inhibited significantly. Moreover, Fo was decreased in Dan598 but increased in Huangzao4 under drought stress. The rise of Fo reflected that the PS II reaction center was destroyed or reversibly inactivated (Ge et al., 2007). The decline of Fo indicated that thermal dissipation that was dependent on xanthophyll cycle enhanced (Demmig et al., 1987). The performance of Fo was consistent with other chlorophyll fluorescence parameters including Fv/Fm, qL and ETR. This further suggested that the functionality of PS II was more limited in non-stay green maize under drought conditions. Zhang et al. (2013) reported that the greater decline of PS II activity and photosynthetic electron transfer was the main reason for the rapid senescence in maize. The decreasing trend of activities of PS II was the same as Pn, suggesting the differential photosynthetic performance of different staygreen maize was due to the activities of PSII. Under drought stress, maize containing stay green trait could maintain higher PSII photochemistry activities to delay senescence than that not containing this trait.

$\mathrm{ABA}$ and CTK are generally considered as two primary hormones to regulate photosynthesis during plant senescence (Yang et al., 2003). In the present study, endogenous hormones were significantly influenced by drought and closely associated with photosynthetic activity. Drought stress significantly enhanced ABA accumulation in roots and leaves, and notably decreased ZR contents in leaves and roots (Fig. 4, 5). The trends of ABA and ZR in roots were the same as those in leaves. ABA changed in a larger extent, while ZR changed in a smaller extent in stay green maize. Furthermore, ABA content in maize leaves showed a negative correlation with with Pn, while ZR was positively related to the investigated photosynthetic parameters of Chl, Pn, Gs, Fv/Fm, qL and ETR (Table 1). These results suggested that both $\mathrm{ABA}$ and $\mathrm{ZR}$ were involved in controlling photosynthetic performance in maize when plants were subjected to drought. Moreover, ZR may play a more important role than ABA in maintaining photosynthetic activities in stay green maize under drought stress. This result agrees with with Yang et al. (2003) who reported that the reduction of CTK induced by drought was closely related with wheat senescence, although ABA could increase drought resistance in plants by enhancing the activity of antioxidant enzymes (Li et al., 2010). Interaction of endogenous hormones may influence physiological processes (Liu et al., 2008). For example, ABA is antagonistic to CTK in regulating stomatal movement (Wang et al., 2004; Tanaka et al., 2006). In this study, ZR/ABA was significantly and positively related with the investigated photosynthetic parameters except Fo, suggesting that the tradeoff between ABA and ZR were also involved in regulating photosynthetic performance. Higher photosynthetic capablity in stay green maize was attributed to higher ZR/ABA when plants subjected to drought stress.

\section{Conclusion}

In conclusion, stay green maize can maintain green leaf area and photosynthetic activities at higher level during the late growth period especially under drought. Both $\mathrm{ABA}$ and CTK were involved in controlling photosynthetic performance, and greater photosynthetic activity of stay green maize could be attributed to higher $\mathrm{ZR}$ and $\mathrm{ZR} / \mathrm{ABA}$ when subjected to drought. In maize 


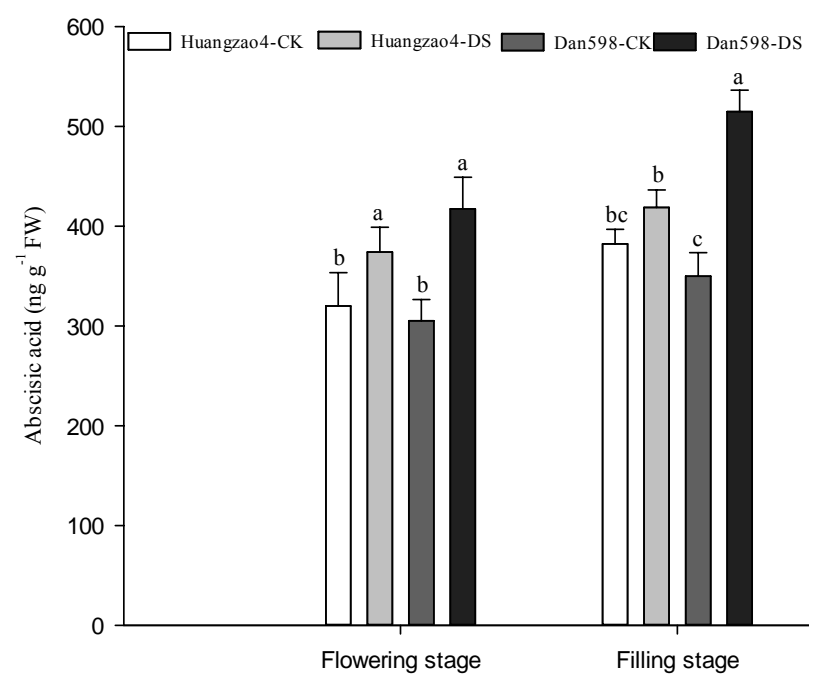

Borrell, A.K., G.L. Hammer and A.C.L. Douglas, 2000. Does maintaining green leaf area in sorghum improve yield under drought? I. Leaf Growth and Senescence. Crop Sci., 40: 1026-1037

Campos, H., M. Cooper, J.E. Habben, G.O. Edmeades and J.R. Schussler, 2004. Improving drought tolerance in maize: a view from industry. Field Crops Res., 90: 19-34

Demmig, B., K. Winter, A. Krüger and F.C. Czygan, 1987. Photoinhibition and zeaxanthin formation in intact leaves: a possible role of the xanthophyll cycle in the dissipation of excess light energy. Plant Physiol., 84: 218-224

Demmig-Adams, B. and W.W. Adams III, 1992. Photoprotection and other responses of plants to high light stress. Annu. Rev. Plant Physiol. Plant Mol. Biol., 43: 599-626

Faraloni, C., I. Cutinob, R. Petruccelli, A.R. Leva, S. Lazzeri and G. Torzillo, 2011. Chlorophyll fluorescence technique as a rapid tool for in vitro screening of olive cultivars (Olea europaea L.) tolerant to drought stress. Environ. Exp. Bot., 73: 49-56

Farooq, M., A. Wahid, N. Kobayashi, D. Fujita and S.M.A. Basra, 2009 Plant drought stress: effects, mechanisms and management. Agron. Sustain. Dev., 29: 185-212.

Ge, L.J., L. Shi, W.B. Gu, Y.D. Tang, J.Z.H. Zhang, C.H.D. Jiang and D.M. Ren, 2007. Photosynthetic Characteristics and the Regulation of Photosystem II Function in Salt-Stressed Sweet Sorghum Seedlings. Acta Agron. Sin., 33: 1272-1278

Huang, R.D., L. Sun, M.J. Xiao, W.J. Xu and Y.F. Zhou, 2009. Physiological and biochemical responses to drought during filling stage in stay green sorghum B35. Acta Agron. Sin., 35: 560-565

Ji, R.P., Y.S. Zhang, L.X. Jiang, S.J. Zhang, R. Feng, P.S. Cheng, J.W. Wu and N. Mi, 2012. Effect of climate change on maize production in Northeast China. Geogr. Res., 31: 290-298

Jia, H.S. and C.M. Lu, 2003. Effects of abscisic acid on photoinhibition in maize plants. Plant Sci., 165: 1403-1410

Ladjal, M., D. Epron and M. Ducrey, 2000. Effects of drought preconditioning on thermo tolerance of photosystem II and susceptibility of photosynthesis to heat stress in cedar seedlings. Tree Physiol., 20: 1235-1241

Li, C.N., M.K. Srivastava, Q. Nong and Y.R. Li, 2010. Mechanism of tolerance to drought in sugarcane plant enhanced by foliage dressing of abscisic acid under water stress. Acta Agron. Sin., 36: 863-870

Liu, R.X., W.Q. Guo, B.L. Chen and Z.G. Zhou, 2008. Effects of nitrogen on the antioxidant enzyme activities and endogenous hormone contents of cotton leaf under drought stress and after soil re-watering during the flowering and boll-forming stage. Acta Agron. Sin., 34: $1598-1607$

Fig. 5: Variations in abscisic acid (ABA) and zeatin riboside (ZR) in roots of stay green maize (Dan598) versus non-stay green maize (Huangzao4) under drought stress. Bars indicate standard deviations. The different letters indicate significant difference at the 0.05 probability level (LSD test) within the same stage. CK, watered normally; DS, drought stress

breeding programs, maize germplasm with stay green trait can be considered as a source to improve drought tolerance.

\section{Acknowledgements}

This experiment was financially supported by the Postdoctoral fund of Shenyang Agricultural University.

\section{References}

Arnon, D.I., 1949. Copper enzymes in isolated chloroplasts. Polyphenol oxidase in Beta vulgaris. Plant Physiol., 24: 1-15

Lu, Y.L., Z.F. Hao, C.X. Xie, J. Crossa, J.L. Araus, S.B. Gao, B.S. Vivek, C. Magorokosho, S. Mugo, D. Makumbi, S. Taba, G. Pan, X. Li, T.Z. Rong, S.H. Zhang and Y.B. Xu, 2011. Large-scale screening for maize drought resistance using multiple selection criteria evaluated under water-stressed and well-watered environments. Field Crops Res., 124: 37-45

Mishra, K.B., R. Iannacone, A. Petrozza, A. Mishra, N. Armentano, G. Vecchia, M. Trtílek, F. Cellini and L. Nedbal, 2012. Engineered drought tolerance in tomato plants is reflected in chlorophyll fluorescence emission. Plant Sci., 182: 79-86

Nawaz, A., M. Farooq, S.A. Cheema, A. Yasmeen and A. Wahid, 2013. Stay green character at grain filling ensures resistance against terminal drought in wheat. Int. J. Agri. Biol., 15: 1272-1276.

Rosenow, D.T., J.E. Quisenberry, C.W. Wendt and L.E. Clark, 1983. Drought tolerant sorghum and cotton germplasm. Agr. Water Manage., 7: 207-222

Tanaka, Y., T. Sano, M. Tamaoki, N. Nakajima, N. Kondo and S. Hasezawa, 2006. Cytokinin and auxin inhibit abscisic acid-induced stomatal closure by enhancing ethylene production in Arabidopsis. J. Exp. Bot., 57: 2259-2266

Tenkouano, A.F., R. Miller, R.A. Frederiksen and D.T. Rosenow, 1993. Genetics of nonsenescence and charcoal rot resistance in sorghum. Theor. Appl. Genet., 85: 644-648

van Kooten, O. and J.F.H. Snel, 1990. The use of chlorophyll fluorescence nomenclature in plant stress physiology. Photosyn. Res., 25: 147-150 
Walulu, R.S., D.T. Rosenow, D.M. Wester and H.T. Nguyen, 1994. Inheritance of stay-green trait in sorghum. Crop Sci., 34: 970-972

Wang, C.T. and S.K. Li, 2010. Assessment of limiting factors and techniques prioritization for maize production in China. Sci. Agric. Sin., 43: 1136-1146

Wang, Q.Q., Y. Chen, H. Xie and J.S. Liang, 2004. Effect of interaction of drought and nitrogen on leaf water potential, somatal conductance and ABA and CTK synthesization in maize. Chin. Agric. Sci. Bull., 20: $20-21$

Yang, J.C., J.H. Zhang, Z.Q. Wang, Q.S. Zhu and L.J. Liu, 2003. Involvement of abscisic acid and cytokinins in the senescence and remobilization of carbon reserves in wheat. Plant Cell Environ., 26 : $1621-1631$

Yang, J.C., J.H. Zhang, Z.Q. Wang, Q.S. Zhu and W. Wang, 2001. Hormonal changes in the grains of rice subjected to water stress during grain filling. Plant Physiol., 127: 315-323

Zhang, J.H., W.S. Jia, J.C. Yang and A.M. Ismail, 2006. Role of ABA in integrating plant responses to drought and salt stresses. Field Crops Res., 97: 111-119
Zhang, R.H., Y.J. Zheng, G.S. Ma, X.H. Zhang, H.D. Lu, J.T. Shi and J.Q. Xue, 2011. Effects of drought stress on photosynthetic traits and protective enzyme activity in maize seedling. Acta Ecol. Sin., 31: 1303-1311

Zhang, S.R., 1999. A Discussion on chlorophyll fluorescence kinetics parameters and their significance. Chi. Bull. Bot., 16: 444448

Zhang, Z.S., G. Li, H.Y. Gao, P. Liu, C. Yang, X.L. Meng and Q.W. Meng, 2013. Changes of photochemistry activity during Senescence of Leaves in stay green and quick-leaf-senescence inbred lines of maize. Acta Agron. Sin., 39: 93-100

Zhou, Y.F., D.Q. Wang, Z.B. Lu, N. Wang, Y.T. Wang, F.X. Li, W.J. Xu and R.D. Hang, 2014. Effects of drought stress on photosynthetic characteristics and endogenous hormone ABA and CTK contents in green-stayed sorghum. Sci. Agric. Sin., 47: 655-663

(Received 28 march 2016; Accepted 29 June 2016) 